\title{
Interaction of central subgerminal ooplasm with the elementary tissues (endophyll, Rauber's sickle and upper layer) of unincubated avian blastoderms in culture
}

\author{
Marc Callebaut*, Emmy Van Nueten, Hilde Bortier, \\ Fernand Harrisson
}

\author{
Laboratory of Human Anatomy and Embryology, \\ RUCA, UA, 171, Groenenborgerlaan, B-2020 Antwerp, Belgium
}

(Received 6 May 1999; accepted 20 September 1999)

\begin{abstract}
By placing a central subgerminal ooplasmic mass over isolated parts (alone or in association) of unincubated avian blastoderms and culture, we obtained an improvement in, or in some cases restoration of normal development. The evolution of small rectangular fragments (isolates) excised from different regions of the unincubated blastoderm was observed in association or not with subgerminal ooplasm. The only type of differentiation that was clearly distinguished in these isolates (taken from the caudocentral area centralis region) was a so-called 'primary neurula' formed by the endophyll and an associated thickened upper layer. In the present study, we also demonstrate that after removal of the area centralis from an unincubated caudal blastoderm quadrant, the upper layer (UL) and endophyll can no longer be restored from the associated subgerminal ooplasm (and form a miniature embryo), as is the case after removal of the endophyll alone. A deep layer (containing the endophyll) reformed during the migration of Rauber's sickle-derived cells into the neighbouring central subgerminal ooplasm only in the presence of the upper layer. This suggests that the upper layer has an early influence on the cells containing the original central deep ooplasm ( $\delta$ ooplasm) to form the endophyll. The present study offers supplementary arguments in favour of the hypothesis that the endophyll is an inductor of preneurulation. (C) Inra/Elsevier, Paris
\end{abstract}

avian blastoderm / central subgerminal ooplasm / endophyll / Rauber's sickle / upper layer

Résumé - Interaction entre l'ooplasme sous-germinal central et les tissus élémentaires (entophylle, croissant de Rauber et ectophylle) du blastoderme non-incubé d'oiseau, en culture. En plaçant de l'ooplasme sous-germinal central sur des parties isolées (seules ou en association) de blastodermes d'oiseau non-incubés, on peut obtenir une amélioration ou même une restauration de leur développement en culture. L'évolution de petits fragments rectangulaires isolés, prélevés à dif-

\footnotetext{
* Correspondence and reprints

E-mail: macal@ruca.ua.ac.be
} 
férents endroits du blastoderme non-incubé, peut ainsi être suivie. Le seul type de différenciation qui peut être clairement observé après culture est la « neurula primaire », formée d'entophylle et d'ectophylle épaissi. De plus, dans la présente étude, nous démontrons qu'après l'excision de l'aire centrale d'un quadrant caudal d'un blastoderme non-incubé, ni l'ectophylle ni l'entophylle ne peuvent se régénérer, même en présence d'ooplasme sous-germinal central. Ainsi un embryon ne sera pas restauré, comme c'est le cas si on enlève seulement l'entophylle. C'est seulement en présence d'ectophylle que l'entophylle se reforme après migration des cellules provenant du croissant de Rauber dans l'ooplasme sous-germinal central. Ceci semble indiquer que l'ectophylle exerce un effet inducteur précoce sur la différenciation entophyllienne des cellules contenant de l'ooplasme central $(\delta)$. La présente étude permet d'apporter des arguments supplémentaires en faveur de l'hypothèse selon laquelle l'entophylle est un inducteur de la préneurulation. (1) Inra/Elsevier, Paris

blastoderme d'oiseau / ooplasme central sous-germinal / entophylle / croissant de Rauber / ectophylle

\section{INTRODUCTION}

Recent studies $[5,6,8,12,13]$ have yielded new data concerning the structures and developmental events in avian intrauterine germs after bilateral symmetrisation and in unincubated eggs. The terminology [13] for the different components of an unincubated quail blastoderm and surrounding structures is represented in figure 1. After $5 \mathrm{~h}$ of incubation, we found that the Anlage of the neural plate in the central part of the upper layer of the area pellucida is already definitively determined [10]. Rauber's sickle divides the area pellucida into a peripheral caudal area marginalis and into an area centralis (figure 1) in which a more or less developed sheet of endophyll can be seen in the caudo-central region. In earlier work [13], we studied the spatial relationship between the different components of the young avian blastoderm after culture in vitro. In the present study, by sandwiching small fragments (isolates) of unincubated quail blastoderms between a vitelline membrane and a coherent mass of central subgerminal ooplasm, we obtained their further normal development after in vitro culture. In this way these fragments were isolated from the influence of the surrounding parts of the blastoderm and followed their own intrinsic developmental pattern. Cells derived from fragments of Rauber's sickles placed in association with central subgerminal ooplasm have the characteristic property to migrate in this ooplasm [7,9]. So nucleated tissues are formed (among which is the endophyll) having the capability to induce a neurogastrulating embryo in the upper layer of a caudal blastoderm quadrant after removal of the whole endophyll sheet [9]. In the present study, in order to see if this can still occur in the absence of UL, we removed the whole area centralis from the caudal blastoderm quadrant in front of Rauber's sickle. To see if the thickened UL, which appears under the influence of an associated endophyll, can still give rise to gastrulation phenomena under the action of Rauber's sicklederived cells, we placed quail blastoderm fragments (containing both endophyll and UL) below the central subgerminal ooplasm in front of a Rauber's sickle and associated caudal part of the blastoderm: no gastrulation phenomena were observed. In a recent study we found evidence that UL from the caudal marginal zone, placed in close apposition with the endophyll, can form (in the absence of the intervening middle layer), a brain Anlage with a large neural groove [15]. In the present study we found more evidence for preneural induction phenomena by the endophyll after the use of other experimental procedures. 


\section{MATERIALS AND METHODS}

We used unincubated chicken and unincubated quail blastoderms presenting a sickle of Rauber, from eggs stored at $15-20{ }^{\circ} \mathrm{C}$ for 1-2 days. After opening of the eggs and removal of the egg white, the egg yolk balls were placed in Ringer solution. The vitelline membrane was sectioned all around the equator of the yolk ball and removed from the yolk by a slow movement in the direction of and with $180^{\circ}$ bending over the germ. In this manner, the chicken blastoderm still adhering to the vitelline membrane and underlying subgerminal ooplasm could usually be separated from the yolk mass. In contrast, in the quail, this procedure was only rarely successful since the unincubated quail blastoderms usually detached from the vitelline membrane. Therefore, to isolate unincubated quail blastoderms, the germ and surrounding yolk must be excised from its egg yolk ball. Thereafter, the yolk, the nucleus of Pander and surrounding ooplasm were progressively removed in order to expose the deep side of the blastoderm. The position and orientation of the different fragments of unincubated quail or chicken blastoderm cultured alone or in association with, completely covered or not covered by the central subgerminal ooplasm, are represented on a schema accompanying the photomicrographs of the explants. The central subgerminal ooplasmic mass, containing the nucleus of Pander in its centre [21], was placed with its superficial side over the deep side of each of the fragments. The subgerminal ooplasmic mass was never lifted out from the liquid of the culture vessel and was moved by a sliding movement over the blastoderm fragments. Some blastoderm fragments were cultured according to the technique described by New [20], others were placed on agar-egg white medium according to the technique of Spratt [23]. This semi-solid medium allowed microsurgery and further culturing on the same substratum. Instead of Petri dishes, the culture vessels described by Gaillard [17] were used, on which an optically flat glass cover was sealed with hot paraffin. Stereomicroscopic polaroid photographs were taken in the same direction at the beginning, during and at the end of the culture period (approximately $24-29 \mathrm{~h}$ of incubation at $38^{\circ} \mathrm{C}$ ). Fixation was performed in a mixture of $0.5 \mathrm{~g} \mathrm{NaCl}, 80 \mathrm{~mL}$ water, $2 \mathrm{~g}$ tricloracetic acid, $4 \mathrm{~mL}$ acetic acid and $20 \mathrm{~mL}$ formalin. After dehydration in an alcohol series, clearing in xylene and embedding in paraffin, the deparaffinised sections were Feulgen stained after Demalsy and Callebaut [16] to identify the origin of the nuclei, using microscope objectives $\times 10$ or $\times 25$. These allowed us to observe the typical central or subcentral chromatin granules of the quail cells $[2,18,19]$ as well as to study their distribution among the chicken cells.

\section{RESULTS}

\subsection{Culture of isolated fragments (quail or chicken)}

Small rectangular blastoderm fragments, with or without covering by the subgerminal ooplasm $(n=22)$ were cultured. Rectangular fragments (approximately $0.4-0.5 \mathrm{~mm}$ in length) were excised from the area centralis (localised centrally from Rauber's sickle) or from the anti-sickle region of unincubated quail blastoderms (figure 2, left). The fragments from the caudocentral region were composed of an upper layer (UL) and underlying endophyll [10] whilst fragments from the craniolateral regions of the area centralis contained mainly if not exclusively upper layer (figure 1). Also fragments from the caudal marginal zone (CMZ) were explanted. The blastoderm fragments were explanted in vitro in two groups. In one group they were placed as such on a chicken vitelline membrane (figure $2 I$ ) and cultured on egg white according to the technique described by New [20]. In a second group a central subgerminal ooplasmic mass (containing the nucleus of Pander in its centre [21]) was placed with its superficial side over the deep side of each of the fragments (figure $2 I I$ ) followed by the same culture procedure. Without covering with the subgerminal ooplasm, the small blastoderm fragments taken from the area centralis or anti-sickle region did not remain flat and rolled up. They did not become adherent to the vitelline membrane during culture. In contrast, the fragments from the caudal marginal zone (schematically represented on figure $2 I$, lower arrow) remained well extended over the vitelline membrane (figure 2IA) and during culture progressively formed a very thin, nearly 

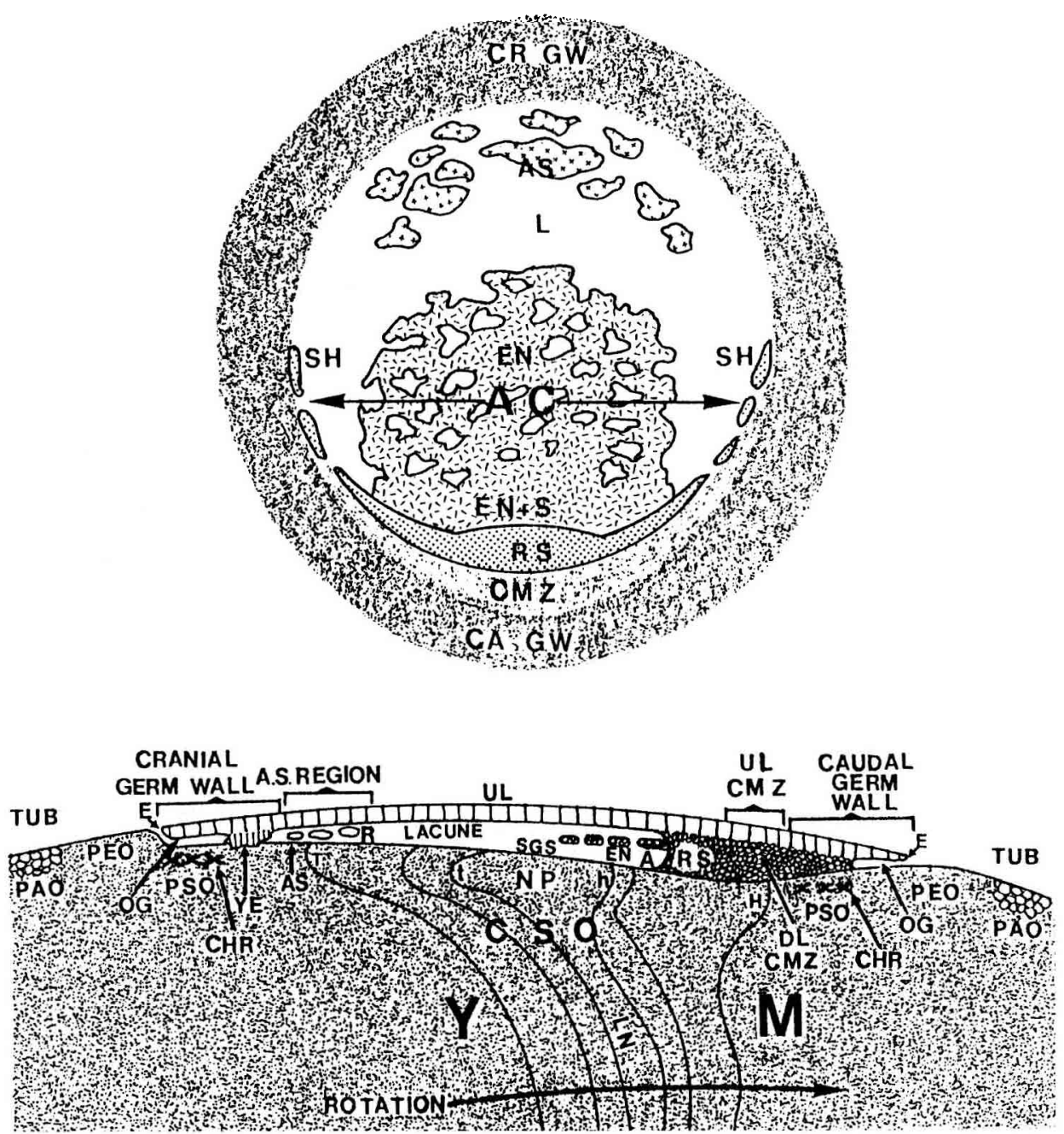

Figure 1. Top: schematic representation of the components of the unincubated quail blastoderm seen from below after removal of the subgerminal ooplasm. CR GW: cranial germ wall; AS: anti-sickle region; L: lacune in the deep layer; EN: incomplete endophyll sheet; EN + S: region of the deep layer where sickle endoblast derived from the Rauber's sickle (RS) meets the endophyll; SH: fragmentary sickle horns; CMZ: more of less transparent caudal marginal zone; CA GW: caudal germ wall. AC: area centralis enclosed by Rauber's sickle and its sickle horns.

Bottom: schematic representation of a mediosagittal section through an unincubated quail blastoderm with surrounding ooplasms after fixation in situ on the egg yolk ball. UL: upper layer; EN: incomplete endophyll layer; RS: Rauber's sickle; UL CMZ: upper layer from the caudal marginal zone; the caudal marginal zone being a more or less transparent part adherent to the caudal peripheral subgerminal ooplasm (PSO) via a deeper part (DL CMZ); SGS: subgerminal space forming a caudal pocket A (axilla shaped) and a cranial recess $\mathrm{R}$ in which free yolk masses or sometimes cells are found forming the anti-sickle (AS); E: edge of the blastoderm; OG: early overgrowth zone; YE: early development of the yolk endoblast, growing into the peripheral subgerminal ooplasm (PSO); CHR: chromosome clusters; PEO: perigerminal ooplasm; PAO: paragerminal ooplasm forming a tubulin (TUB)-rich ring at a distance from the edge of the blastoderm; YM: the voluminous yolk mass of the 
transparent layer adhering to the vitelline membrane (figure $2 I B$ ). After fixation and sectioning, the cultured fragments from the area centralis and anti-sickle region became partially necrotic. The cultured fragments from the caudal marginal zone formed undifferentiated tissue. After covering the blastoderm fragments with central subgerminal ooplasm (represented schematically in figure $2 I I$ ), the fragments usually remained visible by transparency at the start of the culture (figure 2IIA). After culture (figure 2IIB), it was seen that the form and outline of the subgerminal ooplasmic mass had not changed (which seems to indicate the absence of colonisation by nuclei or cells). The borders of the fragment (taken from the centre of an unincubated quail blastoderm), presented a more round aspect than at the start of the culture. After culture and after sectioning, these quail blastoderm fragments presented the aspect of a so-called 'primary neurula' (figure 2IIC). The upper layer had thickened considerably and showed signs of preneurulation $[14,15]$, with vertically elongated cells, nuclei and nucleoli. A primitive streak (PS) was never formed, which can be explained by the fact that no influence of either Rauber's sickle or sickle endoblast had taken place. There was a more or less wide space between this thickened upper layer and the endophyll (figure 2IIC). The endophyll was often present as a larger mass of cells without the spaces and membranous aspect found in a cranial avian endophyllic crescent. In other sections, however, the endophyll cells were also present in the latter more typical form and were connected to the deep side of the thickened upper layer by a small number of very long threads. The thickened upper layer presented some bending in the direction of the endophyll on its borders. This explains the round borders seen on the stereomicrograph after culture (figure 2IIB). The covering of the central subgerminal ooplasm contained no nuclei. Thus, neither the quail upper layer cells nor the quail endophyll cells colonised the ooplasm. This resulted in the absence of morphological changes in the subgerminal ooplasm, as seen on the stereomicrographs (figure $2 I I B$ ). Rectangular fragments of unincubated quail blastoderms taken from peripheral regions of the area centralis covered by subgerminal ooplasm, did not present the typical aspect of primary neurulas, after culture. In the sections, the upper layer was usually flattened and surrounded some deep layer cells which were not recognisable as endophyll cells.

Rectangular fragments of the caudal marginal zone taken from unincubated quail blastoderms and covered by subgerminal ooplasm of chicken (figure 3A), after culture took the aspect of massive round spheres (figure $3 B$ ) in contrast to similar fragments not covered by the subgerminal ooplasm (compare with figure $2 I B$ ). In the sections, no recognisable elementary tissue was discerned in them. They did not colonise the surrounding ooplasm.

When the central subgerminal ooplasm placed over an endophyll-Rauber's sickle complex (as represented schematically in figure $4 A$ and visible on the stereomicrograph of figure $4 B)$ was cultured $(n=5)$, a dense, progressively enlarging $V$-shaped structure starting from Rauber's sickle (figure $4 C$ ) formed. In the sections, large yolk

egg yolk ball in which the eccentricity of the successive yolk layers parallel with the eccentricity in the blastoderm is represented; CSO: central subgerminal ooplasm in which the central nucleus of Pander (NP) [21] is seen; t: toe-shaped and h: heel-shaped part of the nucleus of Pander; T: toe-shaped and $\mathrm{H}$ : heel-shaped part of the surrounding yolk layers as a result of an in utero rotation (the arrow indicates the direction of rotation and compression of the yolk mass under the combined influence of gravity and egg rotation) [5, 7]; LN: bended latebra neck. Note that in contrast to the caudal germ wall, the cranial germ wall is disrupted from the underlying peripheral subgerminal ooplasm $[5,7]$. 

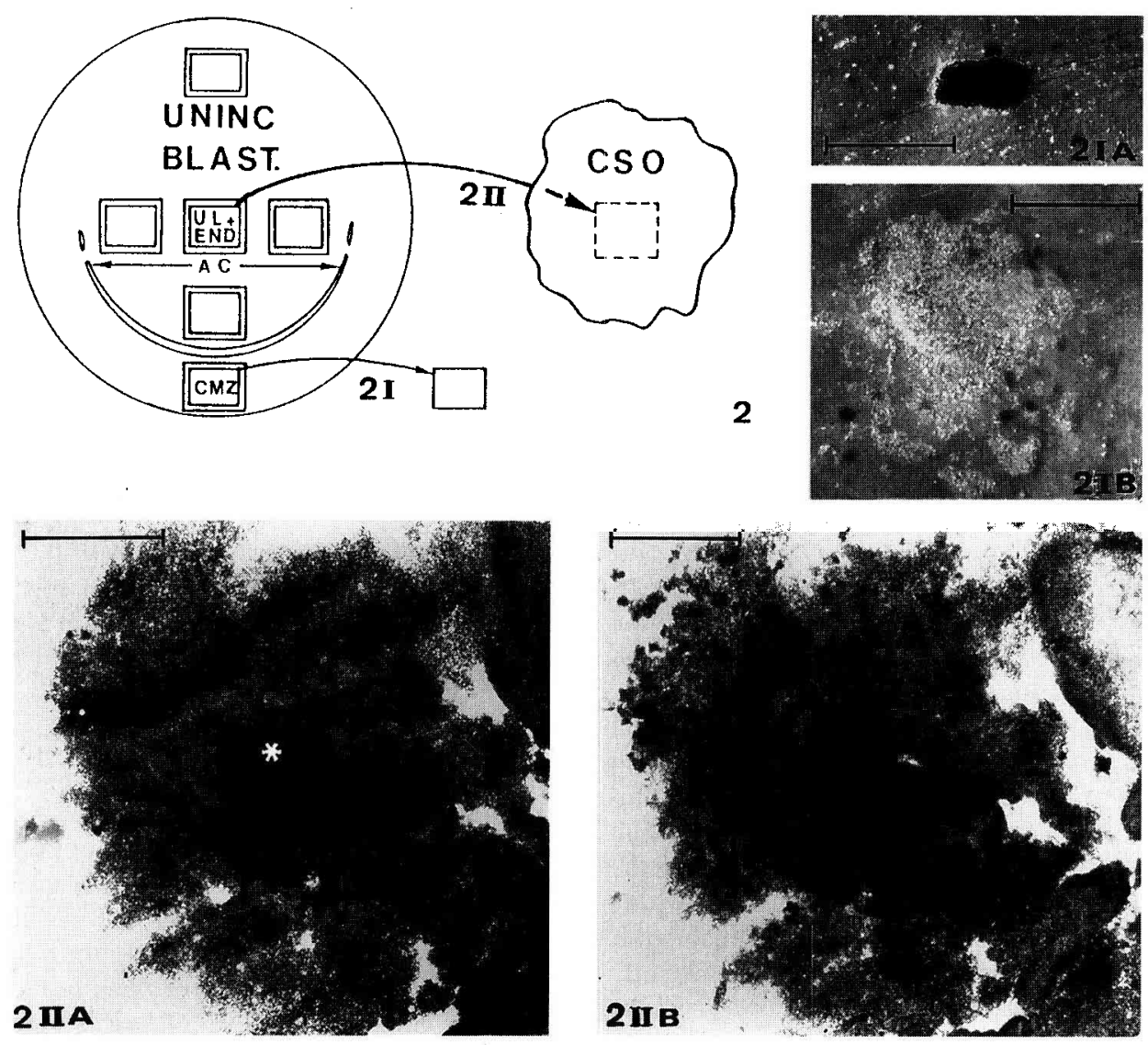

Figure 2. On the left, schematic drawing representing the excision of rectangular fragments taken from different locations from unincubated quail blastoderms. 2I) fragment from caudal marginal zone (CMZ) removed for in vitro culture; 2II) blastoderm fragment composed of upper layer (UL) and endophyll (END), removed from the centrocaudal region and its placing below central subgerminal ooplasm (CSO) for culture in vitro; all the blastoderm fragments are represented with their deep side upwards. 2IA) Stereomicrograph of a rectangular fragment (CMZ) taken from an unincubated quail blastoderm (as schematically represented in 2 by arrow $2 \mathrm{I}$ ), at the start of the culture; bar: $1 \mathrm{~mm}$. 2IB) The same as seen on 2IA after $18 \mathrm{~h}$ of culture; note the flattening and extension of the explant; bar: $1 \mathrm{~mm}$. 2IIA) Stereomicrograph (at the start of the culture) of a rectangular fragment $(*)$ composed of upper layer and endophyll, taken from the centre of an unincubated quail blastoderm (as represented in 2 by arrow 2II); the fragment is visible by transparency through the covering mass of central subgerminal ooplasm (CSO); bar: $1 \mathrm{~mm}$. 2IIB) Stereomicrograph of the same blastoderm fragment as seen in 2IIA, but after $26 \mathrm{~h}$ of culture: the outline and aspect of the covering ooplasmic mass has not changed, whilst the blastoderm fragment presents a round aspect; bar: $1 \mathrm{~mm}$. 2IIC) Section through the quail blastoderm fragment represented in 2IIB: a primary neurula made up of only two layers has formed: a thickened upper layer with (pre)neural plate aspect (NPL) and a deep layer formed by endophyll (END); the covering central subgerminal ooplasm (CSO) is not colonised by quail cells; Feulgen staining; bar: $100 \mu \mathrm{m}$. 
colonising cells with the aspect of junctional endoblast, were observed. This indicates that even in the absence of the upper layer, a junctional endoblast can further develop

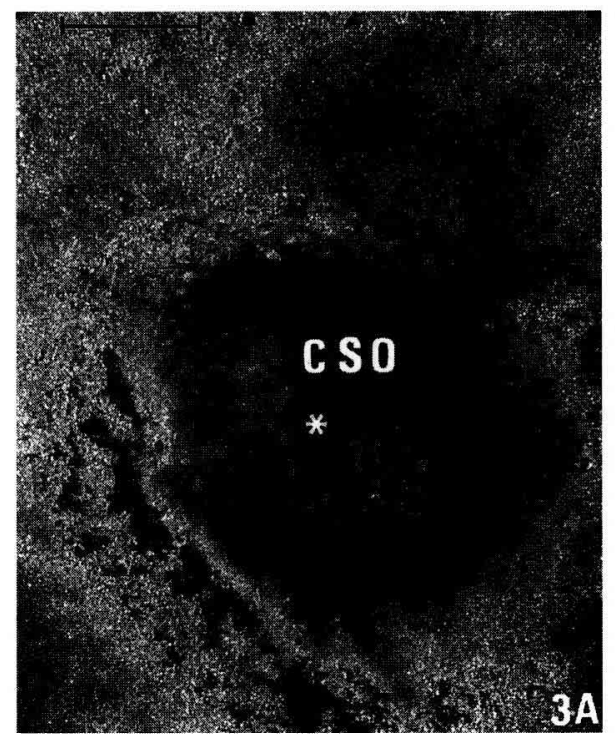

from Rauber's sickle by expansion into the neighbouring ooplasm. An upper layer was not reformed, and a miniature embryo did not develop.

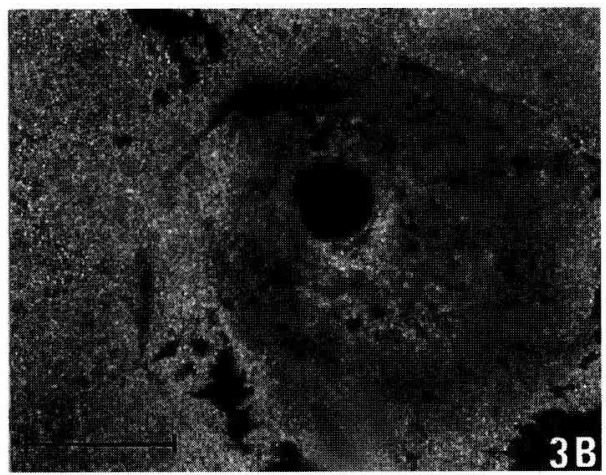

Figure 3. A) Stereomicrograph taken at the start of the culture of a rectangular fragment $\left(^{*}\right)$ of the caudal marginal zone of unincubated quail blastoderm covered with a mass of chicken central subgerminal ooplasm (CSO); bar: $1 \mathrm{~mm}$. B) Stereomicrograph of the same transplant combination as in A, but after $21 \mathrm{~h}$ of culture. Note the dense round aspect of the caudal marginal zone fragment; bar: $1 \mathrm{~mm}$.
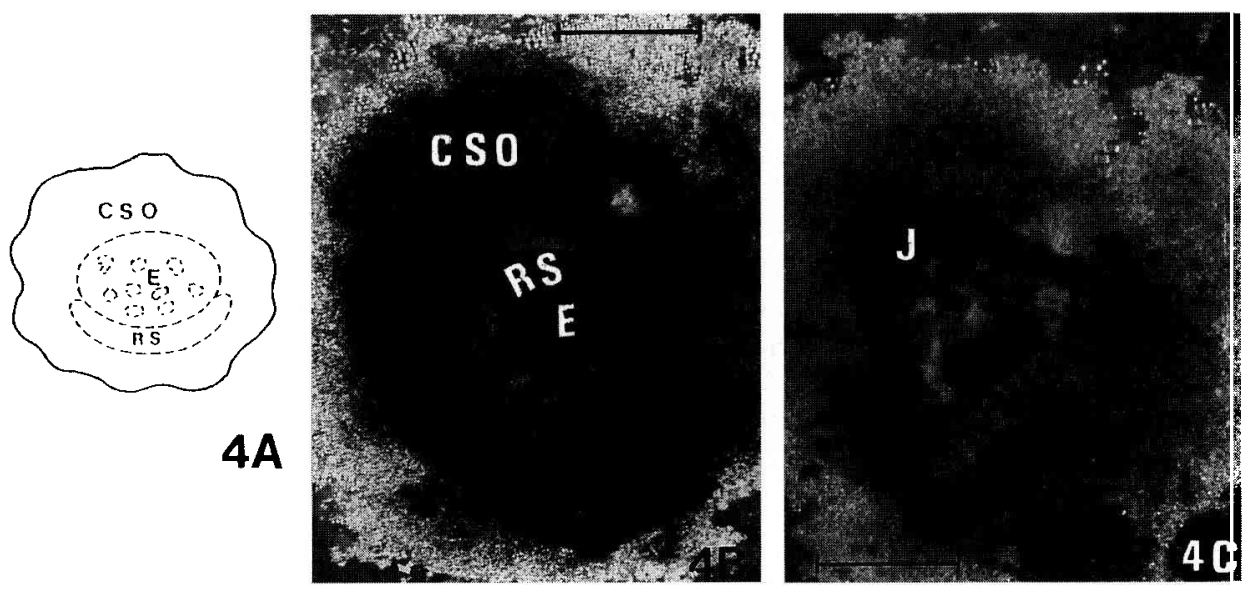

Figure 4. A) Schematic drawing representing the in vitro transplantation of a central subgerminal ooplasmic mass (CSO) over a median fragment of Rauber's sickle (RS) still fixed to its endophyll (E). B) Stereomicrograph of the same association as represented in A, at the start of the culture period; RS: Rauber's sickle quail; E: endophyll still fixed to Rauber's sickle; CSO: central subgerminal ooplasm; bar: $1 \mathrm{~mm}$. C) Stereomicrograph of the transplant of B, after $19 \mathrm{~h}$ of culture; Rauber's sickle has transformed into a more voluminous V-shaped junctional endoblast (J) by partial colonisation of the neighbouring central subgerminal ooplasm (CSO); no UL, nor PS has developed; bar: $1 \mathrm{~mm}$. 


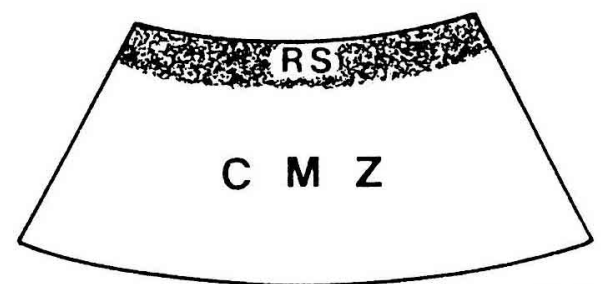

$5 \mathrm{~A}$

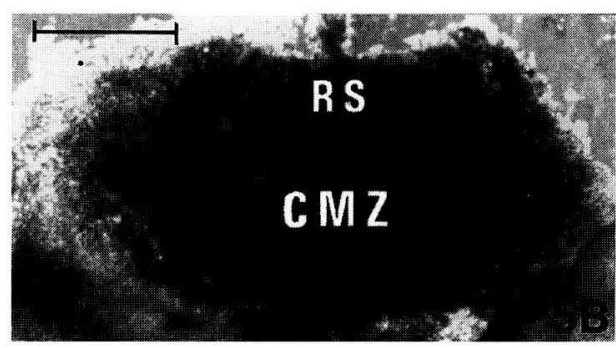

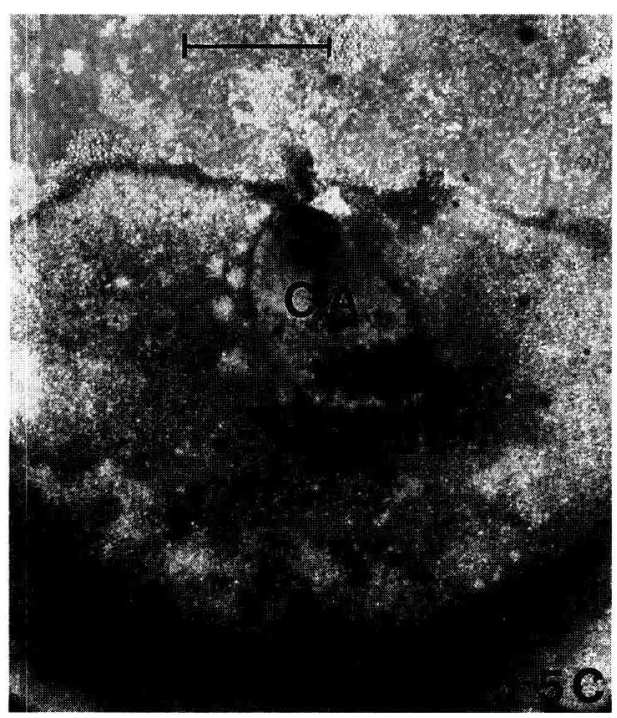

Figure 5. A) Schematic drawing representing a chicken caudal blastoderm quadrant from which the area centralis was completely removed, at the start of the culture. RS: median part of Rauber's sickle still in situ on the caudal part of the caudal blastoderm quadrant; deep side directed upwards; CMZ: caudal marginal zone. B) Stereomicrograph of a chicken explant as represented in A, at the start of the culture; RS: median part of Rauber's sickle; CMZ: caudal marginal zone; bar: $1 \mathrm{~mm}$. C) Stereomicrograph of the explant of $\mathrm{B}$ after $19 \mathrm{~h}$ of culture; note the large development of the area opaca (AO) surrounding the central area (CA) in which no PS nor an embryo is visible (also controlled on sections); bar: $1 \mathrm{~mm}$.

The area centralis was removed from an unincubated chicken caudal blastoderm quadrant $(n=9)$. This procedure is represented schematically in figure $5 \mathrm{~A}$ and visible at the start of the culture on the stereomicrograph (figure $5 B$ ). After culture, these explants (figure $5 C$ ) showed a small central area, surrounded by a broad encircling region (epiboly) of the caudal area marginalis and caudal germ wall. In the sections, no primitive streak, neural plate or embryo could be discerned.

The area centralis was removed from the caudal quadrant of unincubated chicken blastoderms on which a central subgerminal chicken ooplasm was placed $(n=10)$. The procedure is schematically represented in figure $6 A$ and visible at the start of the culture on the stereomicrograph (figure $6 B$ ). In this stereomicrograph, we see the round central subgerminal ooplasm (containing the nucleus of Pander in its centre) overlapping the excised caudal part of the caudal quadrant (containing only Rauber's sickle and tissue behind it). After culture, a centripetal extension of dense material (from Rauber's sickle region) developed in the direction of the centre of the nucleus of Pander, whilst the caudal germ wall expanded caudally and laterally (figure 6C). In the sections, a short primitive streak developed in only one case; in the other nine cases no primitive streak was observed.

\subsection{Culture of associated fragments (quail or chicken)}

The area centralis was removed from the caudal quadrant of an unincubated chicken 

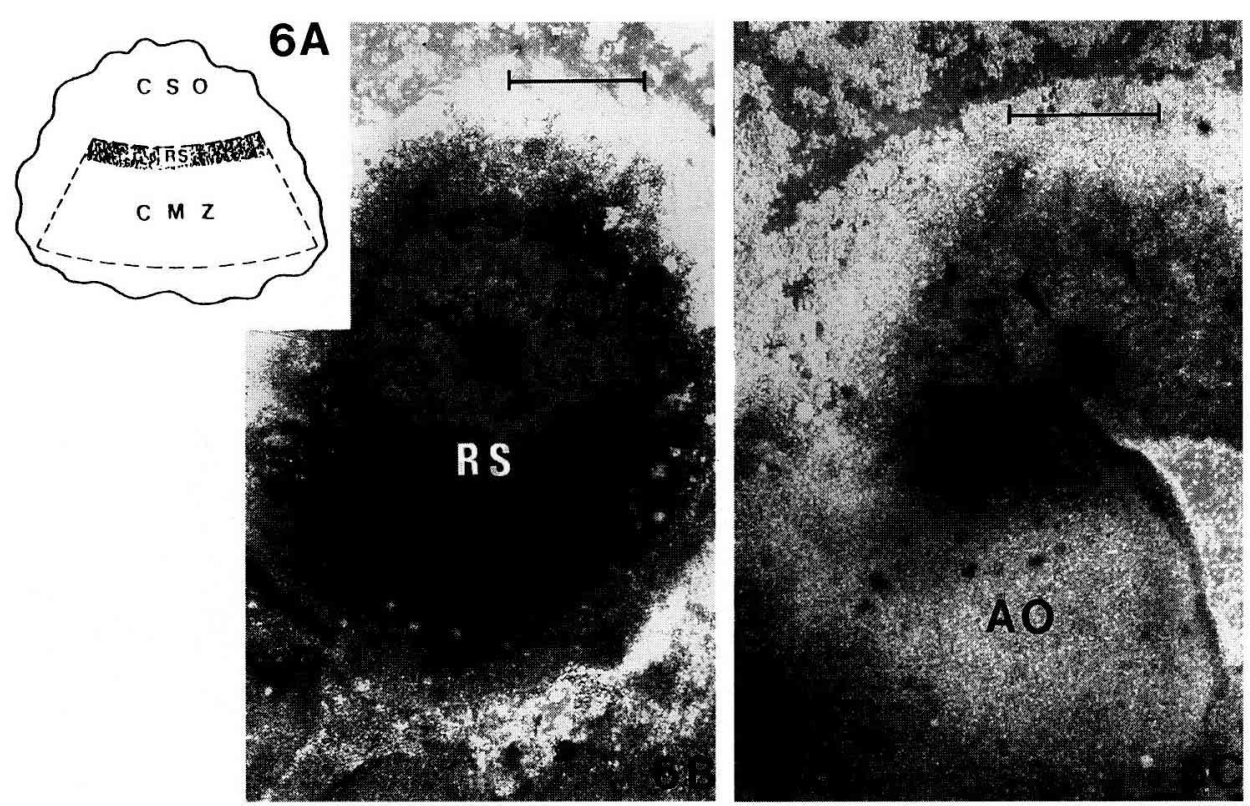

Figure 6. A) Schematic drawing representing a chicken caudal blastoderm quadrant from which the whole area centralis was removed, covered by a mass of central subgerminal ooplasm (CSO); RS: median part of Rauber's sickle still in situ on the caudal part of the caudal blastoderm quadrant; CMZ: caudal marginal zone. B) Stereomicrograph of an association of chicken components as represented in $\mathrm{A}$, at the start of the culture; CSO: central subgerminal ooplasm with, in its centre, the denser part of the nucleus of Pander (indicated by arrowhead); RS: Rauber's sickle: covered by subgerminal ooplasm; bar: $1 \mathrm{~mm}$. C) Stereomicrograph of the same association as in B, after $28 \mathrm{~h}$ of culture. Note the centripetal extension of the tissue material coming from Rauber's sickle region into the direction of the central part of the nucleus of Pander (indicated by arrowhead); AO: area opaca; no embryo develops; bar: $1 \mathrm{~mm}$.

blastoderm on which a central subgerminal ooplasm was placed which also covered a neighbouring central fragment from unincubated quail blastoderm (as represented schematically in figure $7 A)(n=6)$. In figure $7 B$ the different elements of this combination are seen alive at the start of the culture. During the culture, a centripetally directed extension from the Rauber's sickle area progressively grew in the subgerminal ooplasm over the quail graft. After prolonged culture this extension had further grown over the whole quail graft, which remained only faintly visible by transparency (figure 7 ). Finally, the quail blastoderm fragment was completely hidden by tissue (figure $7 D$ ). In the sections, the much thickened quail upper layer (with a preneural plate-like aspect) was seen to be partially recurved at its rims and enclosed the endophyll (figure $7 E, F$ ). Here, also, typical cellular extensions between the endophyll and the inner side of the UL are visible. The whole structure is surrounded by Rauber's sickle-derived cells which had colonised the ooplasm. A primitive streak never formed.

A quail Rauber's sickle fragment was placed at a short distance from a central chicken blastoderm fragment (UL + endophyll) in culture $(n=3)$. This procedure is schematically represented in figure $8 A$ and visible at the start of the culture on the stere- 

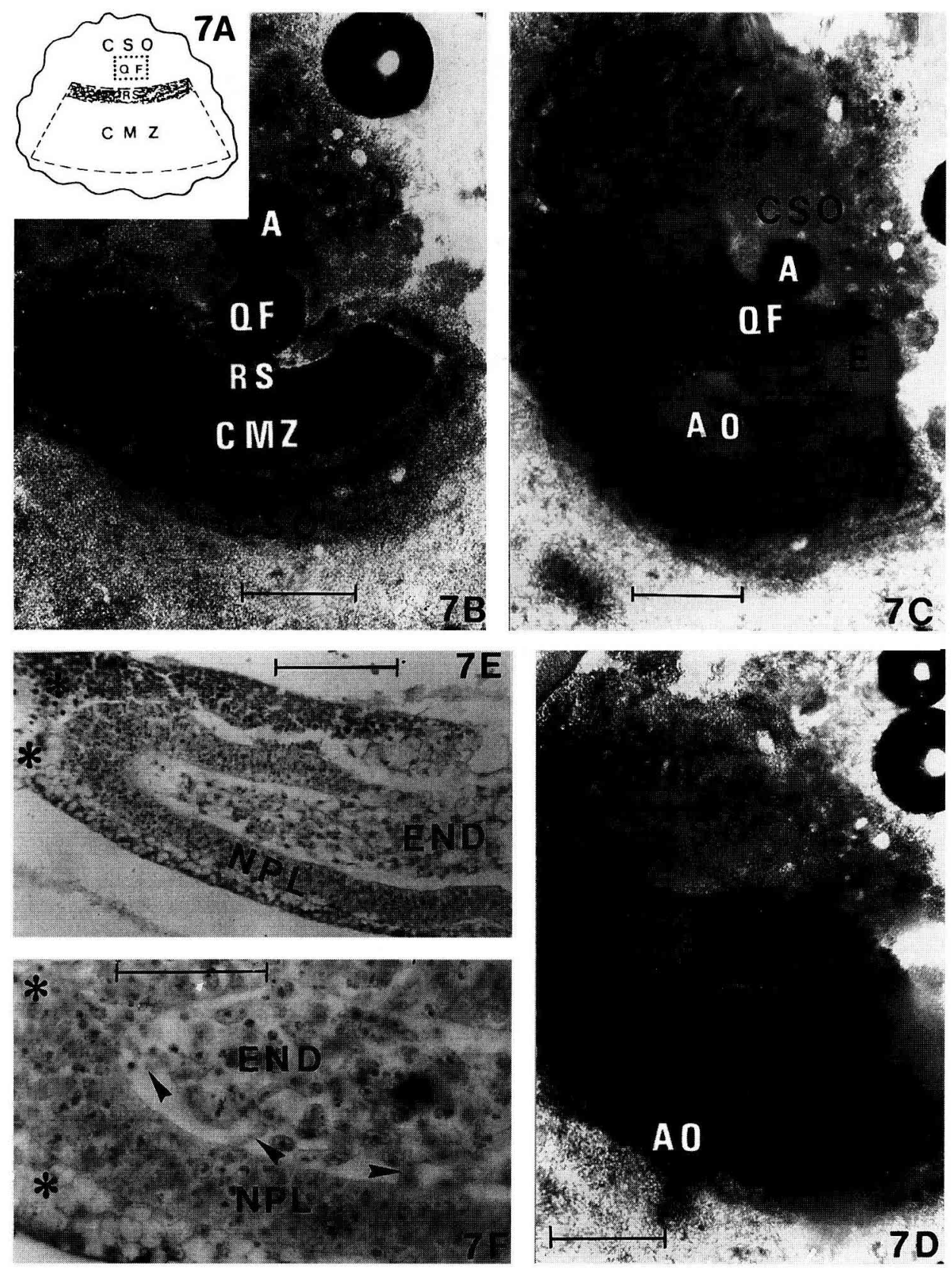

Figure 7. A) Schematic drawing as in figure 6A, but with a rectangular quail blastoderm fragment (QF) taken from the caudocentral region and placed below the central subgerminal ooplasm (CSO) in front of a chicken Rauber's sickle (RS) with its deep side directed upwards. B) Stereomicrograph of a tissue association as represented in A at the start of the culture; QF: quail blastoderm fragment covered by central subgerminal ooplasm (CSO); RS: Rauber's sickle (deep side upwards); CMZ: caudal marginal zone; an air bubble (A) is seen as a round dense sphere in front of the quail blastoderm 


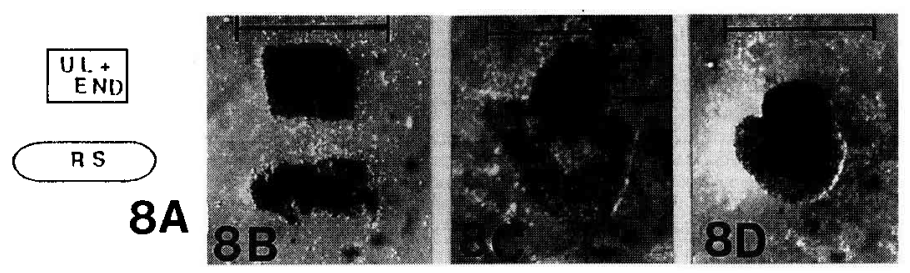

Figure 8. A) Schematic representation of the transplantation of a central chicken blastoderm fragment (UL + endophyll) at a short distance in front of an isolated quail Rauber's sickle fragment (RS), ready for culture. B) Stereomicrograph of a tissue association as represented in A, at the start of the culture; bar: $1 \mathrm{~mm}$. C) Stereomicrograph of the same after $15 \mathrm{~h}$ of culture. The Rauber's sickle has taken a V-shaped aspect close to the central blastoderm fragment; bar: $1 \mathrm{~mm}$. D) Stereomicrograph after $24 \mathrm{~h}$ of culture of the same chimera as in C and B; Rauber's sickle is now in intimate V-shaped contact with the near by part of the central blastoderm fragment; bar: $1 \mathrm{~mm}$.

omicrograph (figure $8 B$ ). Progressively the gap between both was bridged by the Rauber's sickle which developed a V-shaped contact with the blastoderm fragment (figure $8 C, D$ ). In the sections, no PS was seen, only a thickened upper layer and junctional endoblast was observed.

After placing a quail anti-sickle region in front of the concavity of a Rauber's sickle still in situ on the caudal part of an unincubated caudal quail blastoderm quadrant (represented schematically in figure $9 A)(n=9)$. On the stereomicrograph (figure $9 B$ ), the different elements of this combination are visible at the start of the culture period. After $6 \mathrm{~h}$ of culture, a broad centrally directed process starting from Rauber's sickle area was seen (figure 9 C). After $20 \mathrm{~h}$ of culture the volume of the process had regressed (figure 9D). A small denser core was visible in its centre. The surface area of the anti-sickle region in contrast increased and encircled the regressing process. The contact between the two original parts (anti-sickle region and Rauber's sickle region) became progressively closer (which is not always the case); however, the gap between both was still visible. In the sections, a small miniature embryo (with very short primitive streak and neural plate) was seen on sections through the regressing process in only three cases (from a total of nine experiments). It was only when the anti-sickle part largely encircled the process (as in figure 9D) that

fragment; bar: $1 \mathrm{~mm}$. C) Stereomicrograph of associated parts of B after $17 \mathrm{~h}$ of culture; the rim of the chicken Rauber's sickle has grown over the quail blastoderm fragment (QF) as a broad extension (E E) into the central subgerminal ooplasm (CSO); AO: area opaca; $\mathrm{A}$ : air bubble is still visible in front of the quail fragment; bar: $1 \mathrm{~mm}$. D) Stereomicrograph of the same tissue association as in B and C, after $22 \mathrm{~h}$ of culture; the quail blastoderm fragment is now completely hidden by the extension from the chicken Rauber's sickle region; the small air bubble is no longer visible; CSO: central subgerminal ooplasm; AO: enlarging area opaca; bar: $1 \mathrm{~mm}$. E) Section through the quail blastoderm fragment surrounded by central subgerminal ooplasm $\left(^{*}\right)$ colonised by Rauber's sickle cells of $D$. The quail blastoderm fragment forming a 'primary neurula' is composed of only two tissue types: a thickened upper layer forming a (pre)neural plate (NPL) recurved around the endophyll (END); Feulgen staining; bar: $100 \mu \mathrm{m}$. F) Higher magnification of a section through the same tissue association as seen in E; NPL: (pre)neural plate; END: endophyll; cellular extensions between the endophyll and the deep side of the preneural plate are indicated by arrowheads; * surrounding central subgerminal ooplasm colonised by cells derived from the neighbouring chicken Rauber's sickle; Feulgen staining; bar: $50 \mu \mathrm{m}$. 


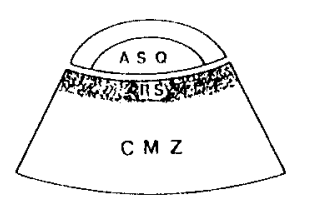

9A

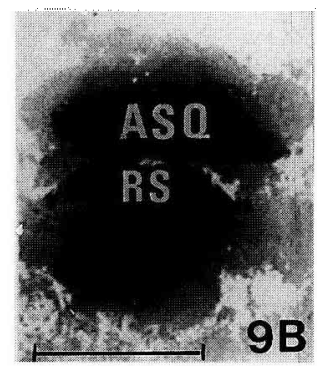

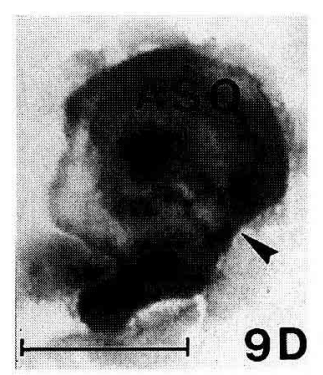

Figure 9. A) Schematic representation of the placing of an unincubated quail anti-sickle region (ASQ) in front of the concavity of a Rauber's sickle (RS) still in situ on the caudal part of the caudal quadrant of an unincubated quail blastoderm (after removal of its area centralis), at the start of the culture period; CMZ: caudal marginal zone; all the tissues are represented with their deep side upwards. B) Stereomicrograph of a tissue association as represented in A at the start of the culture; ASQ: antisickle region of unincubated quail blastoderm; RS: median part of Rauber's sickle; bar: $2 \mathrm{~mm}$. C) Stereomicrograph of the same tissue association as in B after $6 \mathrm{~h}$ of culture: note the broad converging extension of the Rauber's sickle region (RS) into the direction of the anti-sickle region (ASQ); Y: loose yolk mass; bar: $2 \mathrm{~mm}$. D) Stereomicrograph of the same tissue association as in B after $28 \mathrm{~h}$ of culture; ASQ: anti-sickle region of quail encircles a small extension from the original Rauber's sickle region with thickened UL and rudimentary PS (confirmed in the sections); note that both the anti-sickle region and the Rauber's sickle region have not completely fused and are still separated by a cleft (arrow head); bar: $2 \mathrm{~mm}$.

a miniature embryo was formed. A normal arealisation with complete area pellucida around the embryo was never formed.

A central subgerminal ooplasmic mass was placed over a quail anti-sickle region and over the neighbouring concavity of a Rauber's sickle, still in situ on the caudal part of an unincubated chicken blastoderm (schematically represented in figure 10A) $(n=7)$. On the stereomicrograph (figure $1 O B)$, the different elements of this combination were visibly alive at the start of the culture period. After approximately 1 day of culture and partial removal of the covering ooplasm, in five of the combinations, the axis of an embryo could be clearly distinguished (figure 10C). The embryo was much larger than in the cases where no subgerminal ooplasm was placed over it. A sharply defined area pellucida (arealisation) was visible. In the other cases, the embryo was covered with tightly adhering ooplasm and yolk, and remained invisible on the stereomicrographs. After sectioning, how- ever, a normal embryo could also be discerned. By perpendicular serial sectioning of the embryos from which the axis was visible with the stereomicroscope, we could have some insight into the way in which they had developed. In front of the embryo proper, we see that the nucleus of Pander from the subgerminal ooplasm contains nuclei derived from the chicken Rauber's sickle (figure 10D). The upper layer is, however, still present and entirely formed of quail cells. A more caudal transverse section through the cranial part of the embryo (in the region of the endophyllic crescent) shows a deep layer composed of chicken cells which had migrated in the chicken central subgerminal ooplasm (figure $10 E, F$ ). Laterally this deep layer is continuous with fewer cells or no cells containing yolk-rich ooplasm (figure 10E). In the deep layer, there was a decreasing gradient of cellular migration, along with an increasing content of yolk globules, from medial to lateral. In the middle region of the deep layer, the yolk globules seemed to be digested by the pres- 
ence of numerous cells, whilst laterally more and more yolk globules were present. In the deep layer, some quail cells could sometimes be found, probably derived from loose quail cells still adhering to the deep side of the quail upper layer in the anti-sickle region. The upper layer was only composed of quail cells and was somewhat thicker in its middle region (figure $1 O E, F$ ) below the corresponding cell-rich middle region of the neighbouring deep layer. The epigerminal space exterior to the upper layer contained a cast (figure 10E) of material derived from the egg white which had passed through the vitelline membrane [3] during culture. On more caudal transverse sections three zones could be distinguished:

1) a thick median neural plate is visible, parallel and at some distance from a deep layer with an endophyllic aspect as in more cranial sections (figure $10 \mathrm{G}, \mathrm{H}$ );

2) most laterally in the neighbourhood of a deep layer which is very rich in large yolk globules, we found a very thin upper layer (ectoderm);

3) there is also an intermediate deep layer part where some large yolk globules were still present and which contained some chicken cells.

Below the latter, intermediate deep layer zone, a parallel zone in the upper layer exists, which forms a transition zone between the neural plate and the ectoderm. Thus, a clear parallelism in structure and aspect between the three kinds of neighbouring parts of the deep and upper layer exists. The absence of a middle layer (streak derivatives) on all the sections in this region is very obvious. As in isolates of the UL and endophyll, cells and cellular extensions were seen spreading from the deep layer into the direction of the deep side of the neural plate (figure $10 \mathrm{H}$ ). However, they usually did not reach the latter, but this could be due to an artificial increase in space formation between both layers during fixation. More caudal sections, behind the neural plate, showed a well-developed primitive streak-forming area and a primitive streak with a groove (figure 10I) where ingression of quail upper layer cells occurred, forming the mesoderm. In the caudal region of the embryo, the upper layer also only contains quail cells which ingressed through the PS. Here the deep layer was derived from the chicken Rauber's sickle [8], thus only containing chicken cells. It was formed successively from medially to laterally by a thin sickle endoblast, by a thin sheet of transitional endoblast and by a V-shaped junctional endoblast. The successive perpendicular sections through embryos with a stereomicroscopically visible axis thus indicate that the cell containing nucleus of Pander, the endophyllic crescent, the neural plate and the caudocephalic orientation of the PS were formed on line. They are centripetally oriented from a point at the rim of the chicken Rauber's sickle region towards the centre of the nucleus of Pander.

\section{DISCUSSION}

The different behaviour of the isolates (fragments from unincubated quail blastoderms) after being covered with central subgerminal ooplasm and cultured in vitro confirms that there is a fundamental difference in developmental patterning between parts of the area centralis and the caudal marginal zone [10-12]. This seems to be in agreement with studies from Settle [22] and Zagris [24] which demonstrate that haematopoeitic cells develop in isolates of the avian marginal zone even in the absence of gastrulation phenomena. A so-called 'primary neurula' develops after in vitro culture only in fragments taken from the area centralis, where besides UL the endophyll is also present (i.e. in the caudocentral region of the area centralis). No gastrulation occurred since no Rauber's sickle material was included and, consequently, no PS developed. This suggests that the endophyll is a primary inductor for the preneurulation phenomena in the upper layer. Our study 

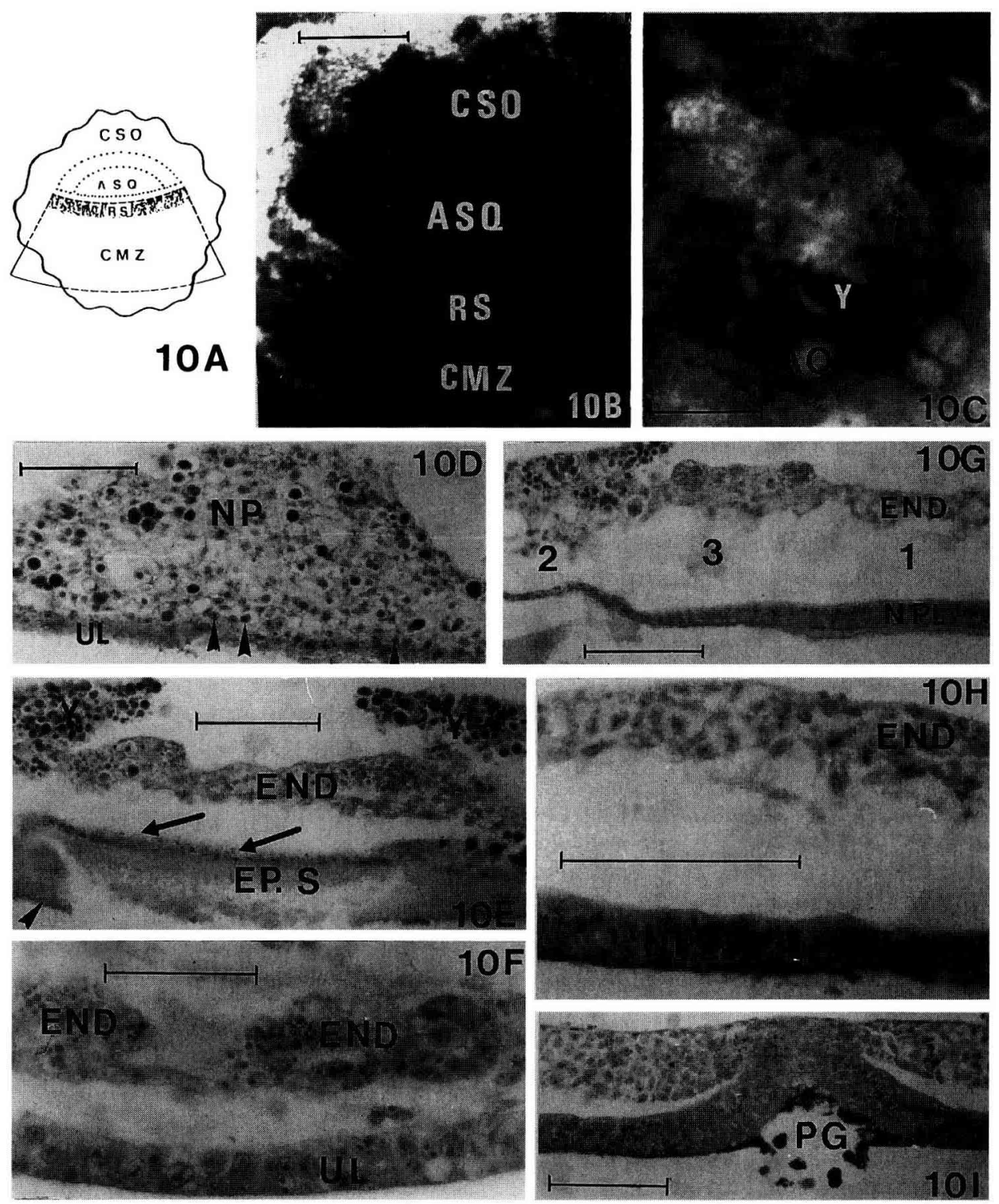

Figure 10. A) Drawing of chimera-association representing the same disposition as in figure $9 \mathrm{~A}$, but after covering with central subgerminal ooplasm (CSO) of chicken: ASQ + RS chick + CSO chick. B) Stereomicrograph of a tissue association as represented in A, at the start of the culture. The tissues are covered by central subgerminal ooplasm (CSO) and remain faintly visible by transparency: ASQ (anti-sickle quail); RS: Rauber's sickle chicken; CMZ: caudal marginal zone chicken; bar: $1 \mathrm{~mm}$. C) Stereomicrograph of the same association of components as in B after $26 \mathrm{~h}$ of culture: a complete embryo has developed with a visible axis (indicated by arrow-head) in a normal area pellucida, sharply delimited from the surrounding area opaca (AO); Y: yolk remnant; bar: $1 \mathrm{~mm}$. D) Section through the colonised central part of a chicken nucleus of Pander (NP) of the central subgerminal ooplasm localised in front of the germ proper of the tissue association of $\mathrm{C}$; narrow 
also demonstrates that once the endophyll has been in the neighbourhood of UL for a sufficiently long time, gastrulation no longer can take place under the influence of Rauber's sickle-derived cells which arrive later (i.e. after colonisation of the neighbouring central subgerminal ooplasm). The fact that only isolates covered with subgerminal ooplasm develop normally in culture can probably be explained by a mechanical holding effect of the apposed ooplasm, since the latter remained unaffected during the culture period (compare figure $2 I I A$ and $I I B)$. In a recent study [15] we found evidence that in the avian embryo, endophyll orients and organises the head region. The avian endophyll therefore seems to be homologous to the cerberus-positive endoblast which induces the overlying ectoblast to differentiate into neurectoderm and directs the orientation of the future head region in Xenopus [1]. Indeed in sections, cerberus mRNA is found in deep yolky cells which by stage 10.5 (Xenopus blastula) are separated from the neurectoderm by a virtual space, called Albert Brachet's cleft [1] which in Xenopus forms long before the bottle cells of the external blastopore lip start ingressing. In the present study, we also observed the stereomicroscopically visible transformation of Rauber's sickle (covered with subgerminal ooplasm) into the more voluminous V-shaped junctional endoblast in the presence of the endophyll but in the absence of the upper layer. Similarly, an upper layer could not be restored from a Rauber's sickle-endophyll complex in the presence of subgerminal ooplasm. Thus, the UL does not seem to be indispensible for the development of the junctional endoblast but the presence of subgerminal ooplasm is required for colonisation by Rauber's sickle cells. Neither the upper layer, nor the endophyll colonises the central subgerminal ooplasm. On the successive stereomicrographs (figure $8 B, C, D$ ), the attraction at a distance exerted by the endophyll-UL association on Rauber's sickle is seen. The rapid cranially directed broad converging outgrowth (already visible after $6 \mathrm{~h}$ ) on the stereomicrograph (figure 9C) of tissue from Rauber's sickle region still in situ on the caudal part of

upper layer (UL) formed of quail cells, (arrowheads) whilst chicken cells have migrated into the nucleus of Pander; numerous dense round primordial yolk globules in a vacuole are still visible in the nucleus of Pander; Feulgen staining; bar: $100 \mu \mathrm{m}$. E) More caudal transverse section through the region of the endophyllic crescent (END) of the embryo of C. In the middle region of the deep layer, the large yolk globules seem to be digested by cellular colonisation, whilst laterally progressively more and more yolk globules are present; the remnants of the uncolonised and undigested ooplasm (filled with large yolk globules) form high walls (Y) laterally; the upper layer (indicated by arrows) only contains quail cells and is somewhat thicker below the endophyll region; EP. S: epigerminal space filled with a cast of albumen-derived material; the arrowhead indicates the vitelline membrane; Feulgen staining; bar: $100 \mu \mathrm{m}$. F) A similar transverse section as in E but at a higher magnification; END: typical endophyll plicae, containing chicken cells and also small yolk granules; UL: thickened upper layer containing only quail cells; Feulgen staining; bar: $50 \mu \mathrm{m}$. G) A more caudal transverse section through the cranial region of the embryo seen in $\mathrm{C}$ shows three distinct parallel regions both in the upper layer as in the neighbouring deep layer: a median thick neural plate (NPL) localised below the endophyll (END); most laterally a very thin upper layer (ectoderm) in the neighbourhood of the thick very yolky and cell poor deep layer; an intermediate transition zone in the upper layer between neural plate and ectoderm parallel and in the neighbourhood of an intermediate deep layer zone which still contains some large yolk globules and only a limited number of chicken cells; Feulgen staining; bar: $100 \mu \mathrm{m} . \mathbf{H})$ Transverse section through the neural plate (NPL) region (composed of quail cells) and neighbouring endophyll region (END) (chicken cells); Feulgen staining; bar: $100 \mu \mathrm{m}$. I) Transverse section through the primitive streak region of the embryo of C; PG: primitive groove; Feulgen staining; bar: $100 \mu \mathrm{m}$. 
the caudal quadrant after removal of the area centralis is remarkable. As in experiments where only the endophyll from the area centralis was removed [9], this process later diminished in volume. Fragments from the UL of the anti-sickle region gave no differentiation, even after covering with the central subgerminal ooplasm. This corresponds to our observations of whole anti-sickle regions covered by central subgerminal ooplasm [14], where, after culture, only extension of the UL was observed. The migration into the nucleus of Pander and surrounding ooplasm of Rauber's sicklederived nuclei seemed to follow a cleavagelike pattern since the general ooplasmic aspect of the nucleus of Pander was still recognisable after culture (figure 10D). The fact, that Rauber's sickle-derived cells and/or nuclei migrate into the central subgerminal ooplasm in a centripetal direction and therefore orient the direction of the caudocephalic axis of the restored embryo has already been suspected in an earlier study [14]. In the present study, this was again observed on stereomicrographs shown in figures $6 C$ and $7 C$ and even more precisely after sectioning of the embryos with fully developed area pellucida (as represented in figure $10 \mathrm{C}$ ). In normal circumstances, in the germ disc of the unincubated avian egg, the ooplasm surrounding the nucleus of Pander separated the latter from Rauber's sickle (figure 1). The present study seems to indicate that the two deep ooplasms in which the nuclei of the blastomeres become lodged, containing different ooplasmic determinants, finally determine the function and further behaviour of these blastomeres. On the one hand, when nuclei and cells migrate in the $\delta$ ooplasm [4] of the nucleus of Pander and become localised in the immediate neighbourhood of the median part of the UL, they transform in deep layer cells with endophyll characteristics. This suggests that the UL has an early inducing effect on the cells, containing original central deep ooplasm. After trypan bluelabelling of the deep paraxial region of the avian oocytal germinal disc followed by a kinetic study in the early embryo, a vertical mixing (at the end of the cleavage stage) of trypan blue-labelled and unlabelled blastomeres occurred. Later most of the trypan blue-labelled cells (containing original deep ooplasm) segregated from the definitive (mainly unlabelled) upper layer cells, settled into the endophyll (early PS stage) and finally also formed primordial germ cells [4]. This segregation and delamination can probably also be explained by interaction of the cells with different determinants in their ooplasm. On the other hand, the original in situ-formed Rauber's sickle cells (containing more peripheral $\beta$ and $\gamma$ ooplasm [4]) give only rise to a localised junctional endoblast (by further migration in the local $\beta$ and/or $\gamma$ ooplasm) and a sickle endoblast which is attracted by the newly formed endophyll. The close association of three elementary tissues (upper layer, Rauber's sickle cells and newly formed endophyll) triggers the formation of a normal embryo [11]. The existence of three different zones in the newly formed deep layer in parallel with three zones in the upper layer, without an intervening middle layer (figure $10 G$ ), suggests a differential vertical influence of the deep layer zones on the corresponding upper layer zones: the median yolk-poor, cell-rich zone (endophyll) with an effect on the thickened neural plate; the lateral, yolk and ooplasm-containing zone on the normal, much thinner ectoderm; the richer intermediate yolk and poorer cell zone on the transition zone between neural plate and ectoderm. Let us note that the thickened median part of the UL (preneural plate: figure $10 G$ ) which a few hours later, transformed into a neural plate could originally only be influenced by a neighbouring endophyll, since the streak derivatives were still localised behind. Our study also indicates that once the upper layer from unincubated blastoderms has been influenced by only endophylls, its fate is determined and it can no longer be influenced by Rauber's sicklederived cells. The present article can be considered as a preliminary study for the even- 
tual use of early molecular markers related to neural induction.

\section{ACKNOWLEDGEMENTS}

The authors wish to thank Mrs V. Van der Stock for skilful technical assistance, Mr F. De Bruyn for photography and artwork and Miss V. De Maere for photography and typing of the manuscript.

\section{REFERENCES}

[1] Bouwmeester T., Kim S., Sasai Y., Lu B., De Robertis M., Cerberus is a head inducing secreted factor expressed in the anterior endoderm of Spemann's organizer, Nature 382 (1996) 595-601.

[2] Callebaut M., Extracorporal development of quail oocytes, Experientia 24 (1968) 1242-1243.

[3] Callebaut M., Autoradiographic demonstration of the penetration of albumen-derived material through the vitelline membrane into the egg yolk, exterior to the avian blastoderm, Poult. Sci. 62 (1983) 1657-1659.

[4] Callebaut M., Ooplasmic localization and segregation in avian blastoderms: fate of the four ooplasms, Arch. Biol. 98 (1987) 441-473.

[5] Callebaut M., Early eccentricity in gravitationally oriented quail germs, Eur. J. Morph. 31 (1993) 5-8.

[6] Callebaut M., Unequal caudo-cephalic ooplasmic uptake and eccentric formation of the subgerminal space below unincubated quail blastoderms presenting a Koller's sickle, Belg. J. Zool. 123 (1993) 107-112.

[7] Callebaut M., Relationship between the avian blastoderm and the subgerminal ooplasm, Eur. Arch. Biol. 105 (1994) 111-123.

[8] Callebaut M., Van Nueten E., Rauber's (Koller's) sickle: the early gastrulation organizer of the avian blastoderm, Eur. J. Morph. 32 (1994) 35-48.

[9] Callebaut M., Van Nueten E., Van Nassauw L., Harrisson F., Restoration of neurogastrulation by subgerminal ooplasm in endophyll free caudal quail blastoderm quadrants, Biol. Jaarb. Dodonaea 63 (1995) 120-132.

[10] Callebaut M., Van Nueten E., Bortier H., Harrisson F., Van Nassauw L., Map of the Anlage fields in the avian unincubated blastoderm, Eur. J. Morph. 34 (1996) 347-361.

[11] Callebaut M., Van Nueten E., Harrisson F., Van Nassauw L., Schrevens A., Bortier H., Avian gastrulation and neurulation are not impaired by the removal of the marginal zone at the unincubated blastoderm stage, Eur. J. Morph. 35 (1997) 69-77.

[12] Callebaut M., Van Nueten E., Bortier H., Harrisson F., Van Nassauw L., Schrevens A., Spatial relationship between endophyll, primordial germ cells, sickle endoblast and upper layer in cultured avian blastoderms. Reprod. Nutr. Dev. 35 (1997) 293-304.

[13] Callebaut M., Van Nueten E., Van Nassauw L., Bortier H., Harrisson F., Only the endophyll-Rauber's sickle complex and not cells derived from the caudal marginal zone induce a primitive streak in the upper layer of avian blastoderms, Reprod. Nutr. Dev. 38 (1998) 449-463.

[14] Callebaut M., Van Nueten E., Harrisson F., Van Nassauw L., Schrevens A., Induction of (pre)gastrulation and or (pre)neurulation by subgerminal ooplasm and Rauber's sickle in cultured anti-sickle regions of avian unincubated blastoderms, Eur. J. Morph. 36 (1) (1998) 1-10.

[15] Callebaut M., Van Nueten E., Harrisson F., Van Nassauw L., Bortier H., Endophyll orients and organizes the early head region of the avian embryo, Eur. J. Morph. 37 (1999) 37-52.

[16] Demalsy P., Callebaut M., Plain water as a rinsing agent preferable to sulfurous acid after the Feulgen nuclear reaction, Stain. Technol. 42 (1967) 133-136.

[17] Gaillard P., Germinal or covering epithelium, Natuurwetensch. Tijdschr. (Gent) 3 de BelgNederl. Cyto-embryol. Dagen, 1949, pp. 5-8.

[18] Koshida Y., Kosin I.L., Intranuclear sex dimorphism in the feathers of six species of galliformes, Cytologia (Tokyo) 33 (1968) 230-240.

[19] Le Douarin N., Barq G., Sur l'utilisation des cellules de la caille japonaise comme marqueurs biologiques en embryologie expérimentale. C. $\mathbf{R}$. Acad. Sci. Paris 269 (1969) 1543-1546.

[20] New D.A.T., A new technique for the cultivation of the chick embryo, J. Embryol. Exp. Morph. 3 (1955) 326-331.

[21] Pander C., Historiam metamorphoseos quam ovum incubatum prioribus quinque diebus subit, FE Nitribitt Wirceburgi, 1817.

[22] Settle G., Localization of the erythrocyte forming areas in the early chick blastoderm cultivated in vitro, Contrib. Embryol. 241, Carnegie Instition of Washington, Washington DC, 1954, pp. 223-237.

[23] Spratt N.T., A simple method for explanting and cultivating early chick embryos in vitro, Science 106 (1947) 452.

[24] Zagris N., Determination of Erythroid cells in unincubated chick blastoderms, Dev. Growth Differ. 27 (1985) 95-100. 